\title{
Silk-Based Nerve Guidance Conduits for Peripheral Nerve Repair
}

\author{
Cuilian Li \\ Guangdong Pharmaceutical University, 280 East Ring Road, Guangzhou Higher Education Mega Center, \\ 510006, Guangzhou City, Guangdong Province, China
}

Keywords: Silk-based nerve guide catheter, peripheral nerve repair, wound.

\begin{abstract}
Trauma-related peripheral nerve injury is a widespread clinical issue that needs to be solved urgently. The complexity of peripheral nerve repair is determined by numerous factors, including a variety of challenging biological repair mechanisms existing in the peripheral nervous system. Autologous peripheral nerve tissue transplantation remains the best clinical treatment for peripheral nerve injury, although there are numerous limitations. In some cases, artificial nerve conduits (NCs) could be a helpful instrumental in the damage repair of peripheral nerve. Recent research results showed that tissue-engineered nerve was adequate essential in repairing peripheral nerve damage, even could overcome some of the limitations of autograft and meet the clinical requirements of long gap nerve repair. Typical components of nerve scaffolds are usually composed of synthetic polymers (e.g., silicone, polyglycolic acid) and natural materials (e.g., chitosan, silk fibroin) alone or a mixture of several. At the same time, diverse manufacturing methods can be used in tissue-engineered NCs, including spinning mandrel technology, sheet rolling, injection-molding, freeze-drying, and electro-spinning. NCs made by tissue engineering methods have attracted widespread attention, and more and more research has focused on the role of silk as a scaffold material in peripheral nerve repair. This review explores the scientific advances in silk-based catheters for peripheral nerve repair.
\end{abstract}

\section{Introduction}

Peripheral nerve injuries have become a major financial burden for the public; the frequency of such injuries has steadily increased over the past few decades representing several hundred thousand cases across the world [1]. Peripheral nerve injury (PNI) is a common condition that for the injured patient results in impaired sensation and reduced motor function of the affection region alongside pain and cold intolerance.[1] After injury, the peripheral nerve obtains certain intrinsic capability to repair itself and normally regenerates at the rate of $1-3 \mathrm{~mm}$ per day. However, the functional recovery of injured peripheral nerve is not always satisfactory. For severe peripheral nerve injury with a large nerve gap, the spontaneous regeneration is largely limited, successful rehabilitation is almost impossible. As a result, the psychological well-being and ability to function in a daily life (e.g., employability, psychological distress, and relationships) are affected.[2] In many cases, such injuries cause lifelong disability due to lack of efficient therapeutic repair measures, particularly in case of severe nerve damages.

Currently, for peripheral nerve injury with no nerve tissue loss or a short nerve gap of less than $5 \mathrm{~mm}$ long, the commonly used surgical technique is end-to-end suturing (nerve coaptation) [3]. insufficient even in the most optimal setting, with a large societal and economical cost.[4] For example, reduced stretching capacity of up to $24 \%$ with end-to-end sutured nerves has been reported, which is due to changes during Wallerian degeneration, intra- and perineural fibrosis, and tissue adhesion [3]. Autologous nerve grafting is the current gold standard for the bridging of nerve gaps that are not amenable to direct suturing, although it is associated with morbidity, loss of sensation, painful neuroma formation and scarring at the donor site [4]. despite several deficiencies such as limited availability and harvesting of functioning nerve, donor site morbidity, time-consuming surgeries, or incomplete recovery.[5] The problems caused by autografts and allografts have led to the use of non-nervous tissue grafts originating from blood vessels, small intestinal submucosa, and 
skeletal muscle tissue [6, 7]. However, the transplantation of autologous nerve graft demands the sacrifice of a healthy donor nerve and the performance of at least two surgeries at both the donor site and the recipient site. Emerging attempts have been made to seek a feasible alternative to autologous nerve graft.

Novel approaches that consider the complex biology underlying PNI are required to physically support tissue growth and elicit desired cellular-specific responses. Biomaterials play a central role in the development of NCs as they significantly influence attachment, proliferation and migration of endogenously regenerating cells [8]. Thus, selection and processing of the biomaterial are critical. A suitable biomaterial should possess good biocompatibility, appropriate degradation properties, and be amenable for controlling secondary NC properties such as pore size, porosity, mechanical strength, and biological functionalization. Neural scaffold also serves as a carrier for cells and biologically active molecules since cells and biochemical cues are generally seeded and incorporated into the scaffold. Therefore, neural scaffold plays a central role in the construction of tissue engineered nerve graft. In particular, silk is a relatively inexpensive biomaterial that has showed promise in the field of nerve tissue engineering and has gained prominence over the last few years due to its physicochemical, mechanical, and biological properties. This review highlights recent progress in the development of natural and synthetic recombinant silks for application in the field of peripheral nerve repair (PNR). Emphasis is put on enhancing the neuroconductive microenvironment of the conduits in terms of physical guidance cues, inner wall, and lumen modification through concentration gradients of peptides and growth factors to direct regeneration. In addition, we introduced the clinical applications of these neural scaffolds and proposed the construction of ideal peripheral nervous system tissue engineering.

\section{Neurobiology and Peripheral Nerve Repair}

Peripheral nerve injuries are common and have a disproportionate impact on young people and the working population. It is estimated that there are more than 1 million new cases of PNI every year worldwide, of which about $40 \%$ occur in Europe. [6-8] The causes of PNI are almost always traumatic, but this may also be iatrogenic or neurological sacrifice during tumor removal. PNI is not temporary, but lifelong injury: It is reported that less than $60 \%$ of people return to work after an injury to the nerves of the upper limbs each year, [2] affecting the prospect of patients returning to work because every 201 patient is susceptible to plexus injury Despite the best microsurgical intervention, functional recovery is always incomplete Therefore, long-term disability of the patient is foreseeable, and an employee's health and living costs are estimated to exceed 50,000 Euros

\subsection{Nerve Biology and Current Treatments}

\subsubsection{Natural Nerve Repair}

Some functional recovery is possible whereby peripheral nerve axons can regenerate; however, the probability of spontaneous recovery decreases as the level of injury increases. Whether crushed or cut, a cascade of cellular events unfolds with extensive changes in the phenotype of injured neurons and Schwann cells distal to the injury that dictates neuronal survival and subsequent regeneration. The remaining surviving axons retract behind the injury front, while the distal segments undergo a process known as Wallerian degeneration. This process, starting 24-48 h after injury, involves fragmentation of the axon and the surrounding myelin. Macrophages infiltrate and engulf the cellular debris during degeneration in a synergetic mechanism along with Schwann cells that aid to clear and prepare the injury site for regeneration. This process may last up to several months, depending upon the length of injury.[9] In the most severe cases, the lack of guidance cues and the presence of fibrin deposition and dense scarring may create physical obstacles to regenerating axons.

This regeneration process is mediated by several biochemical signals, in which the flux of growth factors, which are usually in a healthy state, is impeded when damaged, and the regeneration mechanism is initiated. During this process, samples are drawn from the bulbs at the end of the axon to determine survival time and direction. The presence of Schwann cells provided some of these clues. 
After the injury, Schwann cells lost their unique gene expression patterns [10] and activated repair-related procedures to change from a myelin phenotype to a regenerating state. This includes surface proteins and several neurotrophic factors such as nerve growth factor (NGF), leukemia inhibitory factor (LIF), glial-derived nerve growth factor (GDNF), growth-associated protein GAP-43, and glial growth factor (GGF) Up. Axon elongation and survival of damaged neurons are also promoted in this way. Subsequently, the upregulation of cytokines activates the innate immune response. This allows macrophages to recruit to damaged nerves, promote distal blood flow, and cooperate with Schwann cells to degrade myelin fragments that inhibit axon growth [11]. Macrophages are also attracted to this site by cytokines such as interleukin-6 (IL-6) or leukemia inhibitory factor (LIF) and directly act on neurons to promote axon regeneration. During this repair process, Schwann cells contacted at the distal free axon and continued to proliferate and arranged longitudinally into a solid tubular structure. These are the so-called Bungner bands, which, together with trophic factors secreted by Schwann cells, serve as a pathway for axon regeneration. However, the growth rate of axons is limited to $1-3 \mathrm{~mm}$ per day, and the fact that axons regenerate along the growth path does not determine a successful functional outcome.

\subsubsection{Current Treatments for Peripheral Nerve Repair}

There have been several treatments for nerve reconstruction after segmental defects, and they have been reviewed $[10,12]$. However, where surgery is needed, technology has hardly changed in recent years, and many factors (e.g., age, time, degree of injury, and surgical technique) affect the results. When direct tension-free repair cannot be achieved, an autograft is used as the benchmark. However, the increase in surgical time and lesions in the donor site [12-14] proved the need to find a better neural catheter. Besides, nerve grafts need to be quickly and appropriately sized for effective reconstruction. Allogeneic nerve transplants have also been used to overcome the limitations of autotransplantation, but their use has been affected by host immune rejection. Although alternative tissues have initially successfully filled smaller nerve gaps, allogeneic tissue grafts may not be suitable for supporting nerve regeneration in the event of severe nerve damage due to the lack of appropriate biochemical and topographical elements.

\subsubsection{Clinically Approved Nerve Conduits}

Transplantation of autologous nerve grafts collected from other parts of the body with excellent regeneration effects is now considered the standard gold technique [15]. Besides, its application is mostly limited by the limited number of autologous donor nerves used for implantation, loss of donor site function, structural differences between donor and recipient sites and their possible mismatches, and donor Possibility of forming a neuroma at the site [16]. For example, allogeneic or xenogeneic nerve grafts or other non-neurological tissues (such as veins and muscles) are often accompanied by antigenic and immune problems[15-17].

With the rapid development of tissue engineering and regenerative medicine, tissue engineering nerve grafts have been widely used as potential substitutes for autologous nerve grafts to repair peripheral nerve injuries. A wide range of natural and synthetic polymers have been developed for the manufacture of NCs, some of which have been approved by regulatory authorities for use in the human blood-brain barrier; However, artificial nerve catheters currently on the market have limited functional capabilities for repairing even small nerve gaps [12]. This often leads to complete failure or unsatisfactory clinical outcomes of neural regeneration. According to data from clinical studies in the literature, the neural tube can achieve significant functional recovery in $70 \%$ of patients. Nerve cementation seems inappropriate due to lack of elasticity and collapse of the lumen, because of stiffness and swelling, $[18,19]$ and high incidence of complications, including compression of the device Others, such as SaluBridge, are non-degradable and should be avoided due to chronic nerve compression and fibrosis.

On the other hand, collagen-based nerve catheters, such as NeuraGen, may be an effective treatment option in 43\% of cases; [20] safety and effectiveness have been in the small nerve space of non-critical nerve injury Proven in repair. Tube rigidity and high manufacturing costs, however, are some of their concerns. AxoGen acellular transplant, the only commercially available acellular xenograft and recently approved by the FDA (2010-2013), [20] has been successfully used to repair 
facial nerve defects in humans [21] and hand bridge length $3 \mathrm{~cm}$, and the injection can be restored within nine months of feeling close to normal levels. In rat models, they are superior to other currently available catheters, such as neurons, but have failed to gain an advantage over autograft. Therefore, the repair of severe nerve injury requires not only rough guidance and protection from the surrounding environment but also nutritional information (such as growth factors) and topographical information (guides nano or microstructure) from the nervous system.

\subsection{Tissue Engineering of the Peripheral Nerve: Bioactive Nerve Conduits}

Tissue-engineered nerve grafts are similar to other engineered tissues and bind to scaffolds, cells, biochemical, and physicochemical factors. Tissue-engineered nerve scaffolds (referred to as nerve scaffolds) provide the necessary physical support and guidance for nerve fibers to target tissues or organs. Therefore, tissue engineering is widely regarded as a promising strategy for repairing peripheral nerve injury and the gold standard for future care. It uses the principles of engineering and life sciences to restore and repair tissue function by providing an appropriate physiological environment at the damaged site. A standard method is to culture the cells in vitro on a 3D scaffold and then transport the cells to the desired location in the patient.

Another method is to implant the stent directly in the body, using the host's environmental factors and the active clues of the stent to promote and guide the formation of tissue in the body [22,23]. However, due to the challenging healing process of tissue damage, the requirements of scaffolds for peripheral nerve repair are manifold. For optimal nerve regeneration, the structure must maintain longitudinal strength during repair to prevent loss of continuity. Schwann cells should be promoted to optimize hot and nutrient-inducing cues that attract neurites to the distal nerve and reduce the formation of neuromas while blocking the in-growth of non-nerve tissue that may hinder regeneration. [24] The idea is, therefore, that the development of biologically active neural catheters can mimic the elements of autologous nerve transplantation. Need to promote regeneration-barrier function. The physical integrity of the neural outer membrane. Maintenance repairs, directed guidance cues from endoneurial tubes, a mixture of immune compatible support cells, and availability of blood vessels cluster.

A wide range of materials, including synthetic and natural materials, have been extensively studied as potential candidates for tissue-engineered neural catheters. Synthetic materials are expected to make their performance easy to adjust and control; however, they lack the existence of specific protein binding and cell interaction sites, so their integration with natural tissues is insufficient. Therefore, their biological response must be enhanced; however, the natural spatiotemporal complexity presented by local ECMs has not been well replicated, as mentioned previously, [25] several synthetic polymers have been extensively tested. However, they are limited to small diameter nerves, and only small gap injuries can bridge. Although there is some regeneration, functional recovery is weak, and subsequent surgery must be performed. This is mainly due to poor mechanical properties, lack of cell orientation, or concerns about byproducts and related inflammatory reactions. Also, there is a lack of reliable clinical data. Natural materials have the advantage of conferring biological sites required for protein binding and biological cues that control cell behavior.

Natural scaffolds for peripheral nerve regeneration include decellularized scaffolds for nerve guide ducts (NGCs), glycosaminoglycans such as hyaluronic acid, chitosan, and alginate polysaccharides, collagen, laminin, or gelatin Gel [22,26]. In addition to availability, the main disadvantages of acellular scaffolds are differences in decellularization and post-processing techniques that may lead to incomplete removal of cellular components and damage to microstructures; another issue is that they may serve as a nidus for disease transmission and immune response. On the other hand, glycosaminoglycans, polysaccharides, and protein gels often do not have sufficient mechanical properties to withstand stress. In an aqueous environment, they tend to swell and collapse over time. Cross-linking agents can overcome this problem; however, the clinical application of most ECM components is limited because they usually come from animals or tumorigenic tissues. In terms of neural regeneration, no one seems to have more significant benefits 
than others, and it may be necessary to add more active elements to solve the biological problem of regenerative nerves.

\section{Silk for Peripheral Nerve Regeneration}

Techniques to help nerve regeneration and communicate with neurons require better biomaterial-based bioactive catheters that can both physically support tissue growth and cause the required cell-specific responses.

\subsection{Why Silk is a Suitable Material for Peripheral Nerve Repair}

A variety of other natural proteins and polysaccharides, including alginate, keratin, spider silk protein, and silk fibroin, are also used alone or in combination with other components for neural tissue engineering. Alginate, also known as alginate, is a widely distributed polysaccharide in the cell wall of brown algae. Alginate can promote the vigor and growth of Schwann cells, and promote the germination of neuronal processes in embryonic dorsal root ganglia $[27,28]$. The axon elongation and nerve regeneration were successfully obtained by repairing the $50 \mathrm{~mm}$ sciatic nerve space of cats with two alginate sponges or polyglycolic acid mesh tube patches filled with alginate sponges[29]. The combined use of fibronectin and alginate also achieved better regeneration effects [27,29]. Keratin is an essential fibrous structural protein that exists in human hair and protects cells from damage or stress. In vitro studies have shown that keratin can enhance the attachment and proliferation of Schwann cells. The application of keratin nerve scaffold in vivo can effectively repair $4 \mathrm{~mm}$ nerve defect of mouse tibia [30], 10mm defect of rat sciatic nerve, $15 \mathrm{~mm}$ defect of rat sciatic nerve[31]. The hydrogenated keratin NeuraGen ${ }^{\circledR}$ catheter successfully repaired a 10-mm median nerve defect in cynomolgus monkeys, enhancing motor recovery and nerve regeneration [32].

Spider silk protein is extracted from spider silk. Spider silk protein has excellent tensile strength, good elasticity, and long-term biodegradability. The cultured Schwann cells can quickly adhere to spider silk fibers, making spider silk proteins a competitive natural material for constructing neural scaffolds [33]. The silk protein catheter containing spider silk protein-based Spidrex ${ }^{\circledR}$ fibers supports neurite outgrowth in vitro and repairs the $8 \mathrm{~mm}$ space of rat sciatic nerve in vivo [34]. A nerve scaffold consisting of decellularized vein grafts and spider silk fiber fillings can erect a $20 \mathrm{~mm}$ sciatic nerve space in rats [33] and a $60 \mathrm{~mm}$ tibial nerve space in sheep[35].

In order to obtain medium strength and flexibility, a regenerated spider silk protein fiber scaffold was prepared by electrospinning and post-treated with acetone [36]. The regenerated spider silk protein was mixed with lysine-doped polypyrrole and polylactic acid to make a composite scaffold. The compound nerve duct and nerve growth factor increase the migration of Schwann cells and promote the regeneration of axons, effectively bridging the $20 \mathrm{~mm}$ sciatic nerve space of rats within ten months [37]. The application of silk fibroin in numerically controlled processing has attracted increasing attention [38-40].

Silk fibroin (SF) is a natural protein derived from silkworm cocoons or spiders. Due to its excellent biocompatibility, biodegradability, and mechanical strength, SF has been used as a suture for decades and has recently been used in various tissue engineering applications.[41] With proper physical treatment of silk fibers, water-soluble SF can be extracted and processed into various stent types and shapes; the stent can then be loaded with growth factors and other pharmaceutical substances for regenerative medical applications. The interest in using SF CNC machining has only grown in the past few years.[42] Therefore, few studies have shown that SF neural stem cells can promote axon regeneration in short nerve spaces. Assessed by the total number of axons and the gastrocnemius muscle weight, SF NCs promoted nerve regeneration of more than $8 \mathrm{~mm}$ in the sciatic nerve space in rats. However, these results were still lower than the effects of autograft [43]. Compared with other materials used for CNC processing (collagen, PLG, polylactic-caprolactone), the advantage of SF maybe its slow degradability for several months [44,45] and its affinity for cells, Especially Schwann cells that can survive, adhere and migrate along silk fibers [40]. 


\subsection{Enha ncing Bioactivity of Silk-Based Conduits}

The walls and lumen of the catheter provide attention for the provision of scaffolds: 1) directional neurite growth, Schwann cell migration and phenotypic expression, 2) cell maintenance, and 3) reservoirs of exogenous growth factors.

\subsubsection{Targeted Therapeutics of Silk with Proteins and Growth Factors for Enhanced Repair}

Neurotrophic factors are naturally involved in the survival, growth, and differentiation of nerve cells; they are mainly produced by Schwann cells and play a balancing role in nerve cell regeneration. Given their role in nerve injury and regeneration, [44], their targeted delivery is expected to reduce neuronal death, enhance regeneration, and support Schwann cell populations. This has been confirmed in experimental studies, and some of these biomolecules have been incorporated alone or incorporated into silk-based neural conduits. [40-48] For example, NGF is mixed with RSF solution and cast into a film tube; in vitro experiments have shown that nerve growth factor (NGF) is slowly released over three weeks and maintains its efficacy while significantly increasing the growth of neurites [47].

In another study, the biological activity of codelivery and GDNF and nerve growth factor was evaluated within four weeks by combining growth factor with silk fibroin solution and actually electrospinning the pipeline; [44] in vitro experiments showed enhanced sensory neurons and Motor neuron axon growth rate in chicken embryos and glial cells migrate tightly. In a similar study, NGF and GDNF were mixed into a reef-based solution that was spin-coated into a nerve conduit. It has been reported that the synergistic delivery of these two growth factors within 28 days can synergistically enhance neuronal cell activity compared to the control group; [46] however, the efficacy of growth factors has not been determined.

The effects of loaded growth factors on RSF and RSP nerve ducts were studied in vivo. In 2014, NGF was mixed with rsp-based solution and electrospun into a nerve catheter for implantation into a bridge of $20 \mathrm{~mm}$ gap in the sciatic nerve of rats. [48] Recently, NGF and neurotrophic factor (CNF) were mixed into RSF In solution, it was electrospun into a multichannel nerve catheter [46]. The results showed that, although the results were improved overall, the results showed that most of the loading factors were not released within ten days, and the growth factors were rapidly degraded after the first three days.

In another study, oriented electrospun RSF catheters were made by loading brain-derived neurotrophic factor (BDNGF) and vascular endothelial growth factor (VEGF) into RSF solutions [16]. In vitro experiments have shown that the release of these factors is maintained for only two weeks, while in vivo results of implantation of stents under the skin of adult mice show neovascularization and angiogenesis without chronic inflammation [49]. Although various in vitro and in vivo studies using these growth factors alone or in combination have shown promising results, the use of any of these growth factors has not had a real impact on the clinical treatment of nerve damage, according to the authors It is known that no tests have been conducted at present.

Besides, they may have unpredictable interactions: Excessive supply of growth factors may be detrimental to regeneration, and in order for cells to redifferentiate and remyelination, growth factor levels may need to be gradually reduced over time. Nerve tissue repair is regulated by a multi-step process where multiple growth factors and cytokines work together. These growth factors and cytokines work in a centralized, time-dependent manner, and their effects may depend on many different Elements. An ideal cocktail factor may not be possible, and controlling their restraint attachment and time profile release is still challenging. Therefore, the use of concentration gradients as a guideline has recently received widespread attention. [43-50]

The integration of neurotrophic factors (NTFs), Schwann cells or stem cells, and luminal structures (such as gels, multi-channels, or longitudinally aligned nanofibers) is expected to be a way to improve the performance of NCs. NTFS plays an indispensable role in neuron survival and axon 
regeneration, which is a prerequisite for effective reconstruction of peripheral nerve function [14,15]. Extensively studied neurotrophic factors for peripheral nerve repair include neurotrophic factors [e.g., neurotrophic factors]. Nerve growth factor (NGF), brain-derived neurotrophic factor (BDNF), neurotrophic factor 3, 4, 5 (NT-3, -4, -5)], glial cell-derived neurotrophic factor (GDNF) ( Glial cell-derived neurotrophic factor family ligand member), glial cell growth factor (GGF) (neuregulin family member), neurocytokines [e.g., ciliary neurotrophic factor (CNTF), leukemia inhibitory factor (LIF ) And other cytokines, fibroblast growth factors-1 and -2 (FGF-1 and FGF-2), platelet-derived growth factor (PDGF), and insulin-like growth factor-1 (IGF-1). For detailed information on the complex mechanism of growth factors in nerve growth and repair, readers can refer to related textbooks, such as C. Bell [16]. Put, there are two very significant growth factors for peripheral nerve repair. When NGF and GDNF are applied as a single factor, their spectrums of action are different. Although NGF mainly promotes sensory neuron survival and axonal growth, GDNF is considered to be a critical factor in motor axon regeneration in vitro and in vivo [17]. However, other studies have shown that GDNF also supports the survival and regeneration of sensory neurons [19,20].

Another significant difference between NGF and GDNF is that their effects on axon branches are mainly caused by NGF, while axon elongation is mainly promoted by GDNF [21,22]. Interestingly, recent studies have shown that the optimal combination of GDNF and NGF can produce synergistic effects on axon regeneration in vitro and in vivo [22,23]. DRG neurons co-express TrkA and RET receptors [22,24], which respond to NGF and GDNF, which may explain the observed synergistic effects of axon growth, although the underlying molecular mechanisms are still not fully understood. Taken together, these findings reveal the importance of optimal combinations of multiple growth factors for efficient axonal regeneration.

The use of gradients avoids cell overdose (i.e., by providing continuous dosing over a more extended period, ensuring that cells receive a more appropriate and timely exposure dose, thereby enhancing long-term nerve repair. Concentration gradients are currently being studied in a variety of patterns, shapes, and distributions to guide cells [26]. For example, a concentration gradient of NGF is fixed in a poly (2-hydroxyethyl methacrylate) microporous gel, and its release is maintained for eight days to guide the growth of neurites in vitro [18].

Recently, in 3D printed silicone / methacrylic gel hydrogel catheters, the spatially controlled multi-component gradients of NGF and GDNF were fixed, and their releases have been shown to remain well within one month, despite their Stability has not been determined [25]. In addition to growth factors, peptides, and protein molecules such as neutrophils are also bound together. For example, Shoichet and colleagues first fixed a transparent concentration gradient of NGF and neurotrophin-3 on poly (2-hydroxyethyl methacrylate) and poly (l-lysine) cell-penetrating cells for the first time in 2006. Adhere to the bracket. [20] Since then, researchers have explored several gradient concentrations of ECM molecules, often including a combination of fibronectin and laminin, and the results suggest that they can promote axons to expand along with more complex patterns [21-23,27]. For example, a gradient of surface-bound laminin and short peptide 1 containing the IKKAV sequence proved to be sufficient to guide axonal growth [19]. The delivery of NTFS in NCs can be performed in a variety of ways, for example, loading the NC cavity with a simple NTF solution, a gel matrix containing NTF, slow-release microspheres or nanofibers, embedding NTF into the NC tube wall, or through a micropump or The microinjection port [25] delivers NTFS.

Also, NTFS can also be naturally produced from specialized cells seeded into NCs. Typical cells that pass NTFs are Schwann cells, and adipose-derived stem cells differentiate into Schwann-like cells. In this sense, the term "delivery system" has since been used in any technology and material, including natural and genetically engineered cells, which can be used to make NCs, where they are of interest, that is, locally, to deliver biologically active substances to promote Axons grow. The most promising of these methods are discussed in more detail later in this article. The transmission of NTFS by NCs promotes nerve regeneration but does not improve abnormal axonal growth, resulting in a mismatch in the connections between nerve cells and their surrounding targets [26]. Abnormal axon growth may be caused by several defects, including insufficient NTF dose and release kinetics, the use of a single factor instead of multiple growth factors as a natural effect, and the lack of proper 
guidance at the axon level. There is increasing interest in developing new strategies to integrate polymer-based drug delivery systems into NCs effectively.

Data from these studies indicate that similar strategies can be applied to the silk matrix to further enhance the response of silk-based tubular nerve guide wires [19,22]. However, due to the short half-life of growth factors in the body, implanting growth factors directly onto the surface of nerve ducts may not provide a suitable long-term method of administration [24]. In this regard, a concentration gradient of NGF on electrospun oriented RSF fibers was prepared by embedding growth factors in the spinning solution, and its biological activity was evaluated within five days [28]. It is observed daily, and it is found that the four-step gradient of $209 \mathrm{~m}$ is expanded, compared to only $132 \mathrm{~m}$ per day. Uniform growth factors at different concentrations also spin coaxially with SF and SF, indicating that the growth factor The activity remains useful for a long time and promotes the differentiation of neural stem cells [19]. Arrangements have also been explored for other microparticle-based delivery systems [29] combined with the use of gradients. [19] For example, gdnf-loaded silk microspheres are distributed in a uniform or gradient manner in a silk catheter implanted in a $15 \mathrm{~mm}$ rat sciatic nerve space. [19] The results showed a significant increase in the density of nerve tissue in the GDNF-treated group compared to the empty microsphere catheter. In addition, the density of the neuroendocrine marker PGP 9.5 in the distal end of the GDNF gradient stent catheter was significantly higher than that of the uniformly distributed GDNF stent; [19] its density and number of regenerating axons increased.

\subsubsection{Electrical Stimulation and Inherent Electroactive Moieties in Silk-Based Conduits}

Under normal circumstances, the potential gradient of healthy tissue is minimal, but the potential gradient increases after injury: the epithelial barrier is broken, the potential difference between the epithelium is short-circuited, forming a current flowing to the damaged epithelium, and establishing a lateral electric field [23,31]. These are thought to be the result of passive ion leakage and play a role in controlling and integrating a variety of cellular behaviors such as proliferation, division, migration, and nerve budding. [32] Active exogenous electrical stimulation (ES) attempts to mimic these endogenous electric fields and, due to their basis, promotes neural regeneration as early as 1952. [33] A large number of studies have shown that ES can promote Schwann cell proliferation and neural cell differentiation, Axon growth, and expansion, [23,35], and neurotrophic factor production. [36] Previous studies have shown that cytoplasmic redistribution such as cell polarization and tissue, changes in cell membrane ion current, up-regulation of gene expression, or release of growth-promoting molecules are all affected and regulated by extracellular electrical signals [37-41]. For instance, ES has been found to accelerate, increase neuron (e.g., actin cytoskeleton protein expression and T- $\square$ 1- tubulin) and growth-protein (e.g., GAP-43) [42,43] neuron formation and repair. [44]

Some electroactive materials do exist (Table 6), and it is well known that conductive and piezoelectric substrates can transport ES directly into cells. However, induced ES may have dual effects on tissue repair under different parameters. For example, the size of the applied frequency will affect the density of nerve fibers, indicating that when the frequency is $2 \mathrm{hz}$, the nerve conduction speed is fast, the duration is long, and the axon density is high. When there is no stimulation, the frequency is between 20-200hz. [45] Compared with currents larger than 4ma, the current intensity near 1ma has also been reported to increase the number of axons and blood vessels, which indicates that proper intensity can accelerate the maturation of nerves. However, the too high intensity may hinder the recovery of its function [46,47]. Similarly, a short time delay of applying ES within 1 to 2 weeks after injury has been shown to increase the maturity of neuronal components in rats with sciatic nerve defects [48].

\section{Current Status, Challenges, and Opportunities}

Currently recognized nerve catheters for peripheral nerve repair are generally hollow structures. Due to their limited ability to effectively bridge and repair gaps, only a small percentage of patients with PNI benefit. This highlights the fact that currently available neural catheters are only suitable for 
small injury defects, while tubular structures with other elements (filled catheters, physical guidance cues, growth factors, etc.) may be further improved .

The application of electroactive elements as passive electrical stimulation elements in the stent has broad application prospects and is expected to become an important auxiliary means for nerve regeneration. [28] Studies show that silk can be electrically active / conductive; [9,30,35] However, despite encouraging progress in this area, little research has been done on this active scaffold material. More research is needed to guide the applicability of electroactive / conductive silk-based tissue engineering scaffolds. And their effect on peripheral nerve repair and / or combination with other chemical factors. In particular, the inclusion of carbon-based molecules seems promising, not only to achieve new conductive functions, but also to further adjust the cell-material interaction of the structure based on its high surface area to volume ratio. Much work has focused on assessing the effects (active) after electrical stimulation, but the effect (passive) of introducing an electroactive portion into a stent has not been fully studied. This provides an opportunity for the electroactive scaffold to increase the repair process of damaged nerve tissue by enhancing intercellular communication, regulating growth and cell differentiation without the need for exogenous stimulation. [27-29]

In summary, although there are important studies in the field of silk for nerve repair and new technologies are emerging, more efforts on new strategies will promote silk-based scaffolds that can solve the problem of regenerative neurobiology development of. In addition, more research is needed to overcome existing regulatory barriers related to the use of silk nerve catheters for clinical applications before tissue engineering neural catheter approaches replace the standard for autologous nerve grafts. However, it should be pointed out that the injured site is only one of many neurobiological factors in the process of nerve injury repair, and further strategies should be adopted for patients to fully restore their function (ie, restore function). recovery treatment).

\section{References}

[1] S. Jonsson, R. Wiberg, A. M. McGrath, L. N. Novikov, M. Wiberg, L. N. Novikova, P. J. Kingham, PLoS One 2013, 8, e56484.

[2] C. N. P. Bruyns, J.-B. Jaquet, T. A. R. Schreuders, S. Kalmijn, P. D. L. Kuypers, S. E. R. Hovius, J. Hand Surg. 2003, 28, 28.

[3] R. M. McAllister, S. E. Gilbert, J. S. Calder, P. J. Smith, J. Hand Surg. 1996, 21, 4.

[4] H. E. Rosberg, K. S. Carlsson, S. Höjgård, B. Lindgren, G. Lundborg, L. B. Dahlin, J. Hand Surg. 2005, 30, 35.

[5] D. Grinsell, C. P. Keating, BioMed Res. Int. 2014, 2014, 698256.

[6] P.-N. Mohanna, R. C. Young, M. Wiberg, G. Terenghi, J. Anat. 2003, 203, 553.

[7] K. Bekelis, S. Missios, R. J. Spinner, J. Neurosurg. 2015, 123, 1223.

[8] J. Noble, C. A. Munro, V. S. Prasad, R. Midha, J. Trauma 1998, 45, 116.

[9] J. K. Terzis, D. D. Sun, P. K. Thanos, J. Reconstr. Microsurg. 1997, 13, 215.

[10] B. J. Pfister, T. Gordon, J. R. Loverde, A. S. Kochar, S. E. Mackinnon, D. K. Cullen, Crit. Rev. Biomed. Eng. 2011, 39, 81.

[11] O.K. Muratoglu, S.H. Spiegelberg, J.W. Ruberti, N. Abt, PVA hydrogel, U.S patent 7 (2007) 235,592.

[12] M.F. Meek, J.H. Coert, US food and drug administration/conformit Europeapproved absorbable nerve conduits for clinical repair of peripheral and cranial nerves, Annals 60 (1) (2006) $110-116$. 
[13] A.M. Moore, R. Kasukurthi, C.K. Magill, H.F. Farhadi, G.H. Borschel, S.E. Mackinnon, Limitations of conduits in peripheral nerve repairs, Hand 4 (2009) 180-186.

[14] G.R. Lewin, Y.A. Barde, Physiology of the neurotrophins, Annu. Rev. Neurosci. 19 (1996) 289-317.

[15] S.Y. Fu, T. Gordon, The cellular and molecular basis of peripheral nerve regeneration, Mol. Neurobiol. 14 (1-2) (1997) 67-116.

[16] C. Bell, Chemical Factors in Neural Growth, Degeneration and Repair, Elsevier, Amsterdam, 1996.

[17] K.M. Rich, J.R. Luszczynski, P.A. Osborne, E.M. Johnson Jr., Nerve growth factor protects adult sensory neurons from cell death and atrophy caused by nerve injury, J. Neurocytol. 16 (2) (1987) 261-268.

[18] C.E. Henderson, H.S. Phillips, R.A. Pollock, A.M. Davies, C. Lemeulle, M. Armanini, L. Simmons, B. Moffet, R.A. Vandlen, L.C. Simpson, et al., GDNF: a potent survival factor for motoneurons present in peripheral nerve and muscle, Science 266 (5187) (1994) 1062-1064.

[19] C.R. Matheson, J. Carnahan, J.L. Urich, D. Bocangel, T.J. Zhang, Q. Yan, Glial cell line-derived neurotrophic factor (GDNF) is a neurotrophic factor for sensory neurons: comparison with the effects of the neurotrophins, J. Neurobiol. 32 (1) (1997) 22-32.

[20] P. Leclere, P. Ekstrom, A. Edstrom, J. Priestley, S. Averill, D.A. Tonge, Effects of glial cell line-derived neurotrophic factor on axonal growth and apoptosis in adult mammalian sensory neurons in vitro, Neuroscience 82 (2) (1998) 545-558

[21] G. Terenghi, Histol. Histopathol. 1995, 10, 709.

[22] T. Sedaghati, G. Jell, A. M. Seifalian, in Regenerative Medicine Applications in Organ Transplantation (Eds: J. Lerut, S. Soker, R. J. Stratta), Academic Press, Boston, MA 2014, pp. 799810.

[23] J. W. Tupper, J. C. Crick, L. R. Matteck, Orthop. Clin. North Am. 1988, 19, 57.

[24] M. G. Orgel, Clin. Plast. Surg. 1984, 11, 101.

[25] A. Piskin, B. Z. Altunkaynak, A. Çıtlak, H. Sezgin, O. Yazıcı, S. Kaplan, Neural Regener. Res. 2013, 8, 3410.

[26] S. E. Mackinnon, A. R. Hudson, Plast. Reconstr. Surg. 1992, 90, 695.

[27] B. Schlosshauer, L. Dreesmann, H.-E. Schaller, N. Sinis, Neurosurgery 2006, 59, 740.

[28] C. M. Nichols, M. J. Brenner, I. K. Fox, T. H. Tung, D. A. Hunter, S. R. Rickman, S. E. Mackinnon, Exp. Neurol. 2004, 190, 347.

[29] S. Kehoe, X. F. Zhang, D. Boyd, Injury 2012, 43, 553.

[30] R. A. Weber, W. C. Breidenbach, R. E. Brown, M. E. Jabaley, D. P. Mass, Plast. Reconstr. Surg. 2000, 106, 1036.

[31] S.J. Archibald, J. Shefner, C. Krarup, R.D.Madison,Monkeymedian nerve repaired by nerve graft or collagen nerve guide tube, J.Neurosci. 15 (5 Pt 2) (1995) 4109-4123.

[32] W.W. Ashley Jr., T. Weatherly, T.S. Park, Collagen nerve guides for surgical repair of brachial plexus birth injury, J. Neurosurg. 105 (6 Suppl) (2006) 452-456.

[33] J. Lohmeyer, S. Zimmermann, B. Sommer, H.G. Machens, T. Lange, P. Mailander, Bridging peripheral nerve defects by means of nerve conduits, 78 (2) (2007) 142-147. 
[34] F. Stang, H. Fansa, G. Wolf, M. Reppin, G. Keilhoff, Structural parameters of collagen nerve grafts influence peripheral nerve regeneration, Biomaterials 26 (16) (2005) 3083-3091.

[35] M.C. Lu, S.W. Hsiang, T.Y. Lai, C.H. Yao, L.Y. Lin, Y.S. Chen, Influence of crosslinking degree of a biodegradable genipin-cross-linked gelatin guide on peripheral nerve regeneration, $\mathrm{J}$. Biomater. Sci. Polym. Ed. 18 (7) (2007) 843-863.

[36] W. Sufan, Y. Suzuki, M. Tanihara, K. Ohnishi, K. Suzuki, K. Endo, Y. Nishimura, Sciatic nerve regeneration through alginate with tubulation or nontubulation repair in cat, J. Neurotrauma 18 (3) (2001) 329-338.

[37] X. Wang, W. Hu, Y. Cao, J. Yao, J. Wu, X. Gu, Dog sciatic nerve regeneration across a 30-mm defect bridged by a chitosan/PGA artificial nerve graft, Brain 128 (Pt 8) (2005) 1897-1910.

[38] C. Radtke, C. Allmeling, K.H. Waldmann, K. Reimers, K. Thies, H.C. Schenk, A. Hillmer, M. Guggenheim, G. Brandes, P.M. Vogt, Spider silk constructs enhance axonal regeneration and remyelination in long nerve defects in sheep, PLoS One 6 (2) (2011) e16990.

[39] L. Uebersax, M. Mattotti, M. Papaloizos, H.P. Merkle, B. Gander, L. Meinel, Silk fibroin matrices for the controlled release of nerve growth factor (NGF), Biomaterials 28 (30) (2007) 4449-4460.

[40] Y. Yang, X. Chen, F. Ding, P. Zhang, J. Liu, X. Gu, Biocompatibility evaluation of silk fibroin with peripheral nerve tissues and cells in vitro, Biomaterials 28 (9) (2007) 1643-1652.

[41] M. F. Meek, J.-P. A. Nicolai, P. H. Robinson, J. Reconstr. Microsurg. 2006, 22, 149.

[42] S. Chiriac, S. Facca, M. Diaconu, S. Gouzou, P. Liverneaux, J. Hand Surg. 2012, 37, 342.

[43] J. S. Taras, S. M. Jacoby, Tech. Hand Upper Extreme Surg. 2008, 12, 100.

[44] W. W. Ashley, T. Weatherly, T. S. Park, J. Neurosurg. 2006, 105, 452.

[45] A. Farole, B. T. Jamal, J. Oral Maxillofac. Surg. 2008, 66, 2058.

[46] B. D. Bushnell, A. D. McWilliams, G. B. Whitener, T. M. Messer, J. Hand Surg. 2008, 33, 1081.

[47] A. Mosahebi, M. Simon, M. Wiberg, G. Terenghi, Tissue Eng. 2001, 7, 525.

[48] R. Gaudin, C. Knipfer, A. Henningsen, R. Smeets, M. Heiland, T. Hadlock, BioMed Res. Int. 2016, 2016, 3856262.

[49] S. Gunn, M. Cosetti, J. T. Roland, Laryngoscope 2010, 120, S206.

[50] F. E. Karabekmez, A. Duymaz, S. L. Moran, Hand 2009, 4, 245. 